Original Paper

http://indexmedicus.afro.who.int

\title{
Géomorphologie et productivité des forages dans le nord du bassin du fleuve Comoé en Côte d'Ivoire
}

\author{
Yro Hyacinthe $\mathrm{TIE}^{1^{*}}$, Moussa OUEDRAOGO ${ }^{1}$, Kouakou Lazare KOUASSI ${ }^{2}$, \\ Kamagaté BAMORY ${ }^{1}$, Blaise YAO $^{3}$ et Jean BIEMI ${ }^{3}$ \\ ${ }^{1}$ Université Nangui Abrogoua, 01 BP 10588 Abidjan 01, Côte d'Ivoire. \\ ${ }^{2}$ Université Lorougnon Guédé de Daloa, Côte d'Ivoire. \\ ${ }^{3}$ Université Félix Houphouet Boigny, 08 BP 42 Abidjan 08, Côte d'Ivoire. \\ *Auteur correspondant,E-mail : tieyroh@yahoo.fr ; tieyroh@gmail.com
}

\section{RESUME}

L'objectif de cette étude est de caractériser les aquifères et la productivité des forages dans le haut bassin versant de la Comoé, spécifiquement dans la partie nord de la Côte d’Ivoire. La réalisation de ce travail a nécessité des fiches de 952 forages, des cartes géologiques et la base de données cartographiques de la Côte d'Ivoire. Les épaisseurs d'altération y sont importantes avec $87,92 \%$ des forages supérieurs à la classe des épaisseurs moyennes selon le CIEH. Les forages y sont plus profonds et plus productifs sur les schistes que sur les granitoïdes. La profondeur optimale des forages dans cette zone peut être définie entre 40 et $60 \mathrm{~m}$. Les valeurs de transmissivité varient de $5,59.10^{-6} \mathrm{~m}^{2} / \mathrm{s}$ à $2,01.10^{-4} \mathrm{~m}^{2} / \mathrm{s}$.

(C) 2014 International Formulae Group. All rights reserved.

Mots clés : Fleuve Comoé, bassin versant, aquifère de socle, transmissivité, épaisseur d'altération, forage.

\section{INTRODUCTION}

$\mathrm{Au}$ cours des 35 dernières années, la population africaine, qui était de 413 millions en 1975, est passée à 834 millions (plus de $100 \%$ ) dont 420 millions pour l'Afrique au Sud du Sahara (Traoré, 2010). Cette démographie galopante s'accompagne inéluctablement de la hausse des besoins en eau (agriculture, eau potable). $\mathrm{Vu}$ que la qualité physico-chimique et bactériologique des eaux de surface est souvent douteuse et que les traitements sont onéreux, la recherche de l'eau potable s'est alors orientée vers les eaux souterraines dont la qualité répond généralement aux normes de potabilité de l’OMS (Goné, 2001 ; Kouamé, 2007).

Par ailleurs, les ressources en eau souterraine de la Côte d'Ivoire subissent, de plus en plus, des fluctuations irrégulières dues à l'impact des changements climatiques qui affectent la région soudano-sahélienne depuis le début des années 70 et les pressions anthropiques sur les bassins versants (Savané et al., 2001; Goula et al., 2006; Kouamé, 2007). La partie nord du bassin versant du fleuve Comoé où la pluviométrie est faible (800 mm de pluie par an), connaît beaucoup de problèmes d'approvisionnement en eau. La problématique d'accès à l'eau est donc 
préoccupante pour les populations. En effet, les eaux de surface (rivières et retenues d'eau) tarissent pour la plupart compte tenu de la faible pluviométrie et de l'ensoleillement. Aussi, dans cette région, les campagnes de forage enregistrent-elles un taux d'échec élevé rendant ainsi les conditions de vie des populations difficiles. Dans ces conditions, une maîtrise de l'environnement hydrogéologique et une technologie appropriée pour minimiser les échecs pendant les campagnes pour l'implantation des forages (Kouadio et al., 2008) s'avère nécessaire. L'objectif de ce travail est de caractériser les formations hydrogéologiques afin d'établir un lien entre la géomorphologie et la productivité des forages en vue d'optimiser l'implantation des forages.

\section{MATERIEL ET METHODES Site d'étude}

Le fleuve Comoé est l'un des quatre plus grands fleuves de la Côte d'Ivoire, prenant sa source à Péni, au Burkina Faso. Son bassin versant a également une petite incursion au Mali et au Ghana. Il est le troisième grand bassin versant de la Côte d'Ivoire, du point de vue de la superficie, après ceux du Bandama et du Sassandra, avec une superficie de $78000 \mathrm{~km}^{2}$ (JICA, 2001). L'étude couvre la partie nord du bassin versant de la Comoé qui a pour exutoire la station hydrométrique de Aka-Komoékro (Figure 1). La superficie de ce sous bassin est de $56681 \mathrm{~km}^{2}$, soit $72,6 \%$ de la superficie totale du bassin de la Comoé.

Le régime climatique du bassin étudié est de type tropical humide, particulièrement chaud avec une température moyenne de 28 ${ }^{\circ} \mathrm{C}$ et relativement sec. La pluviométrie y est faible et irrégulière $(800 \mathrm{~mm}$ par an). Le climat est caractérisé par quatre saisons : une grande saison sèche de novembre à février, une grande saison des pluies de mars à juin, une petite saison sèche de juillet à août et une petite saison des pluies de septembre à octobre. Au cours de ces dernières années, la pluviométrie est en baisse et la tendance est à la sécheresse.

Le relief est constitué de petits plateaux plus ou moins cuirassés, de plaines alluviales présentant un modelé plan aux pentes très faibles de part et d'autres de la Comoé, avec un alignement de collines d'altitude moyenne (400 m à $750 \mathrm{~m}$ ) qui se détachent nettement dans le paysage au Centre et au Nord de la zone et qui cassent la monotonie du modelé général (Anonyme, 1998).

Les formations meubles sont constituées par des produits d'altération, d'épandage gravillonnaire, de cuirasse latéritique et d'altérites argileuses dont l'épaisseur peut dépasser $20 \mathrm{~m}$. Le substratum rocheux est constitué de formations du protérozoïque inférieur (Granite à 2 micas, Granite à biotite, Granite monzonitique, Granodiorite) et des formations birrimiennes (Schistes et grauwackes) (Figure 2). Parmi ces formations, le granite à biotite et les schistes sont dominants.

Les sols y sont très variés. En zone de savane, prédominent les sols ferrugineux avec de fréquents affleurements de buttes cuirassées (curasses et carapaces latéritiques). Ce sont généralement des sols remaniés indurés (Touré, 2005) caractérisés par l'existence d'un horizon gravillonnaire renfermant des graviers et cailloux de quartz, des gravillons ferrugineux, des concrétions ferrugineuses et des débris de cuirasse. En zone de forêt, les sols prédominants appartiennent aux sols ferralitiques remaniés, faiblement désaturés et comportant surtout les sols remaniés indurés (sur schiste ou granite) et les sols typiques ou rajeunis (sur granite).

La zone d'étude est très intensément fracturée, le milieu peut être supposé continu et assimilé à un "milieu poreux équivalent" pour le calcul des paramètres hydrodynamiques (Kouamé, 1999). 


\section{Acquisition des données}

Les données cartographiques sont des cartes géologiques à l'échelle 1/200000, qui correspondent aux degrés carrés de Dimbokro, Agnibilékro-Kouamé Dari, Bondoukou, Nassian, Boundiali, Dabakala, Kong, Korhogo, Bouna-Téhini, M'bahiakro et Niellé. Toutes ces cartes ont été obtenues à la Direction de la géologie.

Les dossiers complets de 952 forages ont été utilisés. Des données relatives aux coupes géologiques, les débits et les essais de pompage ont été obtenues à partir des archives des services de l'hydraulique villageoise, principalement dans les Directions Territoriales de l'Hydraulique (DTH).

\section{Calcul de la transmissivité}

L'approche du milieu poreux équivalent a été utilisée pour le calcul des valeurs de la transmissivité. La transmissivité est donnée par la formule suivante :

$$
T=\frac{0,183 Q}{\Delta S} \text { ou } T=\frac{2,3 \cdot Q}{2 \pi \cdot S} \ln \left(\frac{r_{2}}{r}\right)
$$

Où :

$\mathrm{T}$ est la transmissivité en $\mathrm{m}^{2} / \mathrm{s}$; Q le débit du puits en $\mathrm{m}^{3} / \mathrm{s} ; \mathrm{S}$ niveau de rabattement dans le puits de projet en $\mathrm{m} ; \mathrm{r}_{2}$ le rayon d'influence en $\mathrm{m} ; \mathrm{r}$ le rayon du puits de pompage en $\mathrm{m}$. Les données de la remontée des essais de pompage ont été utilisées à cet effet.

\section{Détermination des formations géologiques à haut débit}

Les formations géologiques à haut débit ont été déterminées par la superposition de la carte géologique et la carte de répartition des débits. Cela nous a permis de faire le lien entre les formations géologiques et les différentes classes des débits. Les formations géologiques seront alors classées en fonction des classes de débits.


Régon du NZi Comoé Région des Savanes Région de la valée du
Bandama

Région du Zanzan
4. Station hydrométrique

Cours d'eau temporare

$\checkmark$ cours d'eau permanent

Limce de la zone d'étude

Figure 1 : Localisation du site d'étude. 


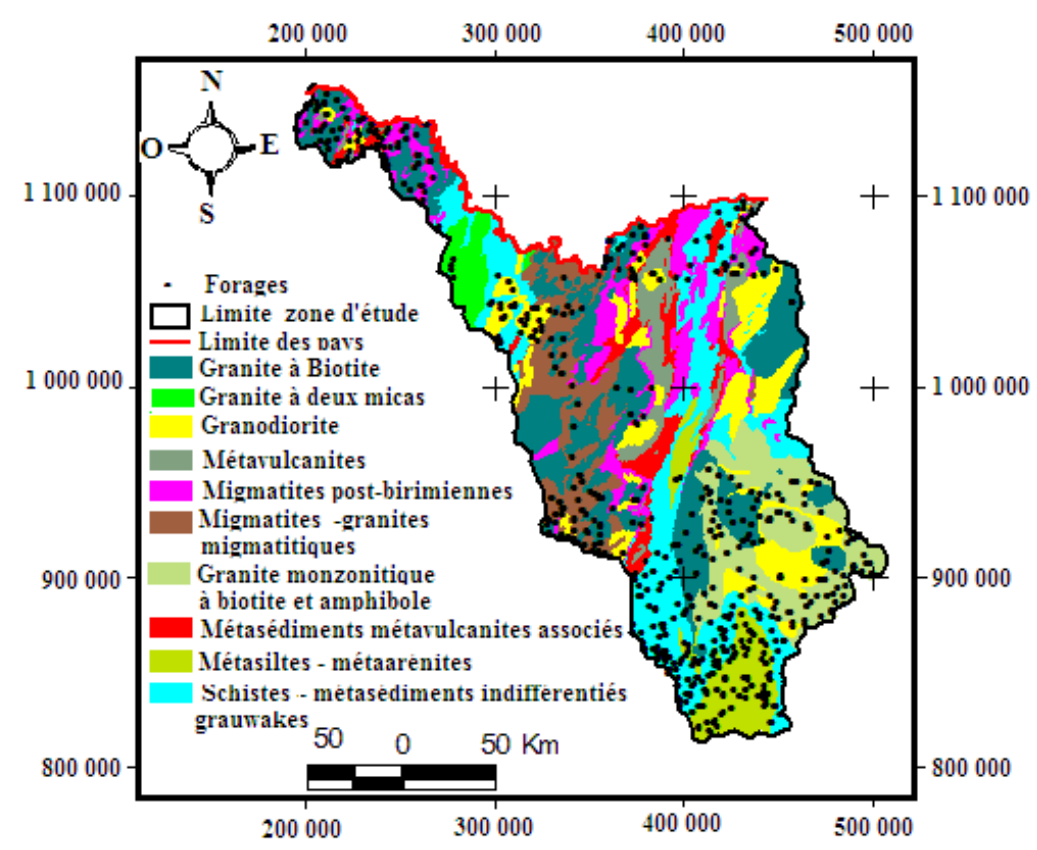

Figure 2 : Distribution des forages dans les formations géologiques de la zone d'étude.

\section{RESULTATS}

\section{Epaisseur d'altération}

La Figure 3 donne une illustration des épaisseurs d'altération de la zone étudiée. Les épaisseurs d'altération sont classées en fonction des cinq classes d'épaisseurs d'altération définies par le CIEH (Comité International des Etudes Hydrauliques) allant de la très faible à la très forte en passant par la faible, la moyenne et la forte (Tableau 1).

A ces classes définies par le CIEH, il a été ajouté une classe dite exceptionnelle car les forages qui la composent ont une profondeur d'altération au-delà de la définition du CIEH. En effet, près de $10 \%$ des forages ont enregistré une épaisseur d'altération supérieure à $70 \mathrm{~m}$.

Dans leur majorité, soit $65,44 \%$, les forages appartiennent aux classes forte et très forte épaisseur d'altération. La classe de l'épaisseur moyenne représente 22,48\%. Les épaisseurs de moyenne à forte concernent donc $87,92 \%$ des forages.

Les faibles épaisseurs se rencontrent au Nord-Est dans la région de Bouna et au NordOuest dans la région de Ferkessédougou tandis que les épaisseurs les plus fortes se rencontrent principalement dans les régions de Bondoukou, Dabakala et Nassian (Figure 3).

\section{Influence de la lithologie sur l'épaisseur des altérites}

L'analyse du Tableau 2 montre que les profondeurs sur schistes sont globalement plus importantes que les profondeurs sur granitoïdes car les classes de profondeurs supérieures à $50 \mathrm{~m}$ sont plus importantes sur les schistes que sur les granitoïdes. Sur les schistes, ces profondeurs ont une fréquence de $86 \%$ pendant que sur les granitoïdes, la fréquence est de $63 \%$.

Par ailleurs, en utilisant la classification des épaisseurs d'altération du $\mathrm{CIEH}$, on se rend compte que les classes de moyenne à très forte profondeur sont prédominantes autant au niveau des granitoïdes que des schistes avec une légère prédominance des schistes pour la classe très forte (Tableau 2).

On remarque que la classe $25-40 \mathrm{~m}$, qui est la classe des fortes épaisseurs, est prédominante pour les deux formations 
géologiques, avec néanmoins une proportion plus élevée sur les granitoïdes. Par contre, les schistes dominent dans la classe très forte (Figure 4).

\section{Profondeur des forages}

Les profondeurs des forages de la zone d'étude varient de $10 \mathrm{~m}$ à $110 \mathrm{~m}$ (Tableau 3). Les forages des classes 40-50 m, 50-60 m, 60-70 m et 70-80 m sont les plus nombreux avec respectivement des fréquences de $19,89 \% ; 23,44 \% ; 17,53 \%$ et $14,09 \%$. La classe la plus prépondérante est celle des 50$60 \mathrm{~m}$ avec plus de $23 \%$ des forages. Par ailleurs, les forages de plus de $80 \mathrm{~m}$ de profondeur ont une fréquence de $16,77 \%$.

On observe que les forages des classes 40-50 m et 50-60 m cumulent plus de $44,33 \%$ pendant que les classes $50-60 \mathrm{~m}$ et $60-70 \mathrm{~m}$ cumulent $40,97 \%$. Ainsi, 85,3\% des forages sont entre $40 \mathrm{~m}$ et $90 \mathrm{~m}$ de profondeur.

\section{Arrivée d'eau}

La Figure 5 présente la répartition des arrivées d'eau par classe de profondeur. A travers cette figure, on constate que quatre classes présentent des fréquences élevées des arrivées d'eau. Ce sont les classes 20-30 m, 30-40 m, 40-50 m et 50-60 m. Parmi celles-ci, les classes 20-30 m et 50-60 m présentent les mêmes fréquences $(15,04 \%)$ alors que les deux autres classes présentent $25,92 \%$ pour les $30-40$ m et $24,35 \%$ pour les $40-50 \mathrm{~m}$.

\section{Productivité des forages selon la profondeur}

Les débits ont été répartis par classe de profondeur et un débit moyen a été calculé par classe. Néanmoins, deux débits exceptionnels supérieurs à $100 \mathrm{~m}^{3} / \mathrm{h}$ ont été supprimés de la liste pour éviter d'introduire des biais.

Le graphique de la Figure 6 montre que les bons débits sont obtenus dans les profondeurs de $30 \mathrm{~m}$ à $90 \mathrm{~m}$, avec un maximum pour la tranche de $40 \mathrm{~m}$ à $60 \mathrm{~m}$. On remarque que les classes $30-40 \mathrm{~m}$ et $100-110$ $\mathrm{m}$ ont pratiquement le même débit moyen. Mais ce dernier est biaisé par un débit exceptionnel de plus de $80 \mathrm{~m}^{3} / \mathrm{h}$.
L'analyse de la répartition des débits par rapport à la profondeur dans les différentes classes fait ressortir également que les profondeurs $40-50 \mathrm{~m}$ et $50-60 \mathrm{~m}$ ont les meilleurs débits. En effet, dans ces classes, les débits allant de 2 à $4 \mathrm{~m}^{3} / \mathrm{h}$ et de 4 à $10 \mathrm{~m}^{3} / \mathrm{h}$ qui sont classés comme moyens et forts par le CIEH ont la plus grande fréquence. La mise en évidence de la relation entre les profondeurs des forages et les débits montre également que les bons débits sont obtenus entre $30 \mathrm{~m}$ et $90 \mathrm{~m}$ de profondeur avec un maximum entre 40 et $60 \mathrm{~m}$ (Figure 7). Il ressort de cette analyse que la profondeur optimale des forages dans cette zone peut être définie entre 40 et $60 \mathrm{~m}$ pour les forages d'hydraulique villageoise et hydraulique villageoise améliorée.

\section{Productivité des forages selon les formations géologiques}

Le Tableau 4 présente la productivité des forages en fonction des différentes formations géologiques du substratum sousjacent. Il ressort de l'analyse de ce tableau que les débits importants (forts et très forts selon le $\mathrm{CIEH}$ ) sont obtenus sur les schistes et sur les granitoïdes. En effet, ceux-ci enregistrent des fréquences de $35 \%$ contre $23 \%$ pour les granitoïdes.

\section{La transmissivité}

Les valeurs de transmissivité, calculées à partir des données d'essai de pompage à l'aide de la méthode de Cooper Jacob, varient de $5,59.10^{-6} \mathrm{~m}^{2} / \mathrm{s}$ à $2,01.10^{-4} \mathrm{~m}^{2} / \mathrm{s}$ (Tableau 5 ). Les valeurs inférieures à $10^{-5} \mathrm{~m}^{2} / \mathrm{s}$ ont une fréquence de $24 \%$. Celles comprises entre $10^{-5} \mathrm{~m}^{2} / \mathrm{s}$ et $10^{-4} \mathrm{~m}^{2} / \mathrm{s}$ ont une fréquence de $52,75 \%$ et les valeurs supérieures à $10^{-4} \mathrm{~m}^{2} / \mathrm{s}$ sont de $23 \%$.

La carte de la Figure 8 montre que les valeurs élevées de transmissivité sont localisées dans la région de Tafiré, Nassian, M'bahiakro et Dabakala. La zone de Ferkessédougou au Nord enregistre les valeurs moyennes et le reste de la zone d'étude a des valeurs faibles de la transmissivité. 
Tableau 1 : Classification des épaisseurs d'altération de la zone selon les critères du CIEH.

\begin{tabular}{lcccccc}
\hline Classes & Très faible & Faible & Moyenne & Forte & Très forte & Exceptionnelle \\
\hline Epaisseur d'altération & $<10 \mathrm{~m}$ & $10-15 \mathrm{~m}$ & $15-25 \mathrm{~m}$ & $25-40 \mathrm{~m}$ & $40-70 \mathrm{~m}$ & $>70$ \\
Nombre & 36 & 79 & 214 & 238 & 291 & 94 \\
Pourcentage & 3,78 & 8,30 & 22,48 & 25,00 & 30,57 & 9,87 \\
\hline
\end{tabular}

Tableau 2 : Répartition des forages par classe d'épaisseur d'altération selon le substratum.

\begin{tabular}{|c|c|c|c|c|c|c|c|}
\hline \multicolumn{2}{|l|}{ Classes } & Très faible & Faible & Moyenne & Forte & Très forte & \multirow{2}{*}{ Total } \\
\hline \multicolumn{2}{|c|}{ Epaisseur d'altération } & $<10 \mathrm{~m}$ & $10-15 \mathrm{~m}$ & $15-25 \mathrm{~m}$ & $25-40 \mathrm{~m}$ & $40-70 \mathrm{~m}$ & \\
\hline \multicolumn{2}{|c|}{ Granite à biotite } & 38 & 54 & 90 & 145 & 74 & 401 \\
\hline \multicolumn{2}{|c|}{ Granite discordant } & 36 & 19 & 30 & 17 & 41 & 143 \\
\hline \multicolumn{2}{|c|}{ Granite à micas } & 10 & 10 & 5 & 11 & 2 & 38 \\
\hline \multicolumn{2}{|c|}{ Migmatites et gneiss } & 1 & 5 & 0 & 1 & 1 & 8 \\
\hline \multicolumn{2}{|c|}{ Schistes birimiens (flysch) } & 5 & 2 & 15 & 31 & 20 & 73 \\
\hline \multicolumn{2}{|c|}{ Schistes volcano sédimentaires } & 56 & 10 & 55 & 54 & 114 & 289 \\
\hline \multicolumn{2}{|l|}{ Total } & 146 & 100 & 195 & 259 & 252 & 952 \\
\hline \multirow{2}{*}{ Granitoïdes } & Effecti & 85 & 88 & 125 & 174 & 118 & 590 \\
\hline & Pourcentage & 14,41 & 14,92 & 21,19 & 29,49 & 20,00 & 100 \\
\hline \multirow{2}{*}{ Schistes } & Effectif & 61 & 12 & 70 & 85 & 134 & 362 \\
\hline & Pourcentage & 16,85 & 3,31 & 19,34 & 23,48 & 37,02 & 100 \\
\hline
\end{tabular}

Tableau 3: Répartition des forages en fonction des classes de profondeur.

\begin{tabular}{lccc}
\hline Classe de profondeur $(\mathbf{m})$ & Nombre de forages & Pourcentage des forages & Pourcentage cumulé \\
\hline $00-10$ & 2 & 0,22 & 0,22 \\
$10-20$ & 2 & 0,22 & 0,44 \\
$20-30$ & 16 & 1,72 & 2,16 \\
$30-40$ & 57 & 6,13 & 8,29 \\
$40-50$ & 185 & 19,89 & 28,18 \\
$\mathbf{5 0 - 6 0}$ & $\mathbf{2 1 8}$ & $\mathbf{2 3 , 4 4}$ & $\mathbf{5 1 , 6 2}$ \\
$60-70$ & 163 & 17,53 & 69,15 \\
$70-80$ & 131 & 14,09 & 83,24 \\
$80-90$ & 96 & 10,32 & 93,56 \\
$90-100$ & 41 & 4,41 & 97,907 \\
$100-110$ & 19 & 2,04 & 100 \\
Total & $\mathbf{9 3 0}$ & $\mathbf{1 0 0 , 0 0}$ & $\mathbf{1 0 0}$ \\
\hline
\end{tabular}

\section{DISCUSSION}

Dans le nord du bassin versant de la Comoé, les épaisseurs d'altération sont comprises entre $25 \mathrm{~m}$ et $70 \mathrm{~m}$. Ces épaisseurs d'altération appartiennent aux classes forte et très forte épaisseur d'altération selon la classification du Comité Inter africain des Etudes Hydrauliques (CIEH). Ce résultat rejoint ceux des auteurs antérieurs. En effet, selon Touré (2005), la zone d'étude appartient au socle précambrien ivoirien caractérisé par des granitoïdes et des schistes. Les travaux de Biémi et al. (1997) ont montré que les profils d'altération sur granitoïdes ont des épaisseurs variant de 0 à $50 \mathrm{~m}$ et sont moins importants que ceux développés sur des terrains schisteux (0 à $100 \mathrm{~m})$. 
Tableau 4 : Répartition des forages par classe de débit par rapport aux formations géologiques.

\begin{tabular}{lccccc}
\hline Formation géologique & $\begin{array}{c}\text { Débit très } \\
\text { fort }\end{array}$ & $\begin{array}{c}\text { Débit } \\
\text { fort }\end{array}$ & $\begin{array}{c}\text { Débit } \\
\text { moyen }\end{array}$ & $\begin{array}{c}\text { Débit } \\
\text { faible }\end{array}$ & $\begin{array}{c}\text { Débit très } \\
\text { faible }\end{array}$ \\
\hline Granite à Biotite & 9 & 14 & 25 & 33 & 19 \\
Granite à deux micas & 2 & 0 & 1 & 1 & 1 \\
Granodiorite & 3 & 15 & 13 & 19 & 10 \\
Métavulcanites & 1 & 0 & 2 & 5 & 0 \\
Migmatites post birimiennes & 1 & 3 & 8 & 16 & 9 \\
Migmatites anciennes - granites migmatitiques & 1 & 5 & 14 & 20 & 19 \\
Granite mozonitique à biotite et amphibole & 4 & 13 & 19 & 20 & 32 \\
Métasédiments - métavulcanites associés & 0 & 2 & 7 & 6 & 8 \\
Métasiltes - métaarénites & 4 & 20 & 27 & 23 & 20 \\
Schistes - grauwakes - métasédiments indifférenciés & 13 & 35 & 35 & 28 & 23 \\
\hline
\end{tabular}

Tableau 5 : Répartition des transmissivités en fonction des classes.

\begin{tabular}{lccc}
\hline Transmissivité T & Moyenne & Pourcentage (\%) & Ecart-type \\
\hline $\mathrm{T}<10^{-5}$ & $5.59 .10^{-6}$ & $24.18 \%$ & $2.13198 .10^{-6}$ \\
$10^{-5}<\mathrm{T}<10^{-4}$ & $4.47 .10^{-5}$ & $52.75 \%$ & $2.98184 .10^{-5}$ \\
$\mathrm{~T}>10^{-4}$ & $2.01 .10^{-4}$ & $23.08 \%$ & $1.03 .10^{-4}$ \\
\hline
\end{tabular}

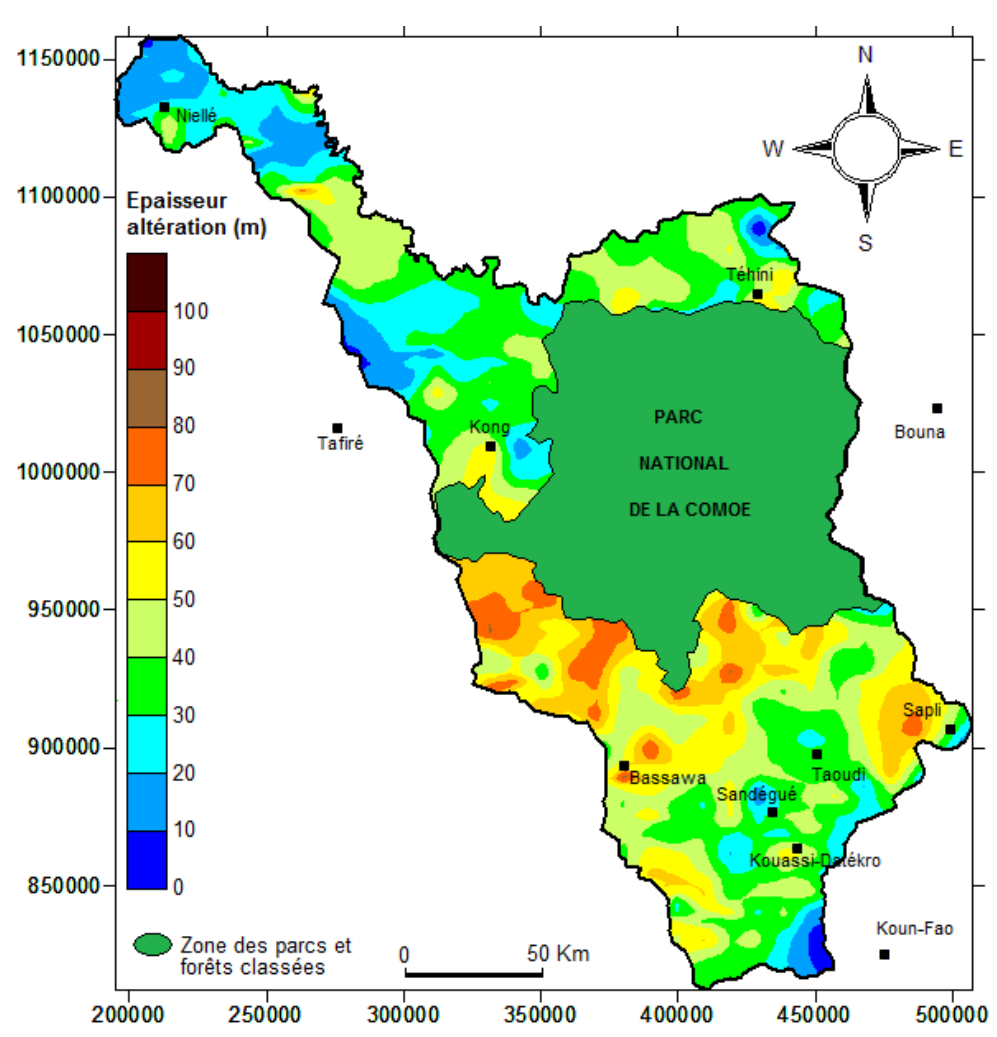

Figure 3 : Carte des épaisseurs d'altération. 




Figure 4 : Evolution des épaisseurs d'altération par classe et par substratum.

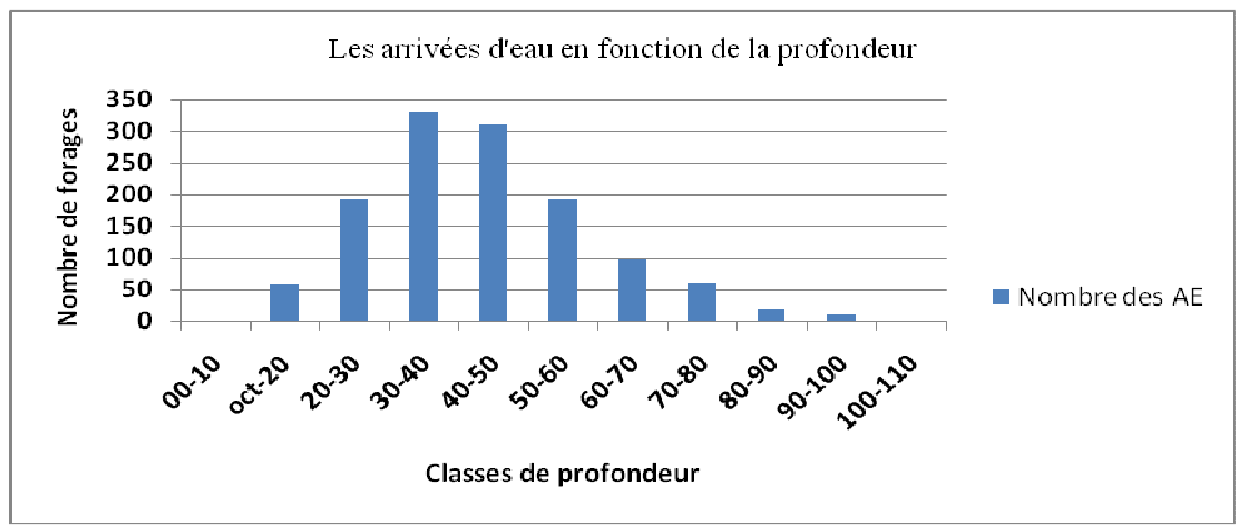

Figure 5 : Répartition des arrivées d'eau en fonction de la profondeur.

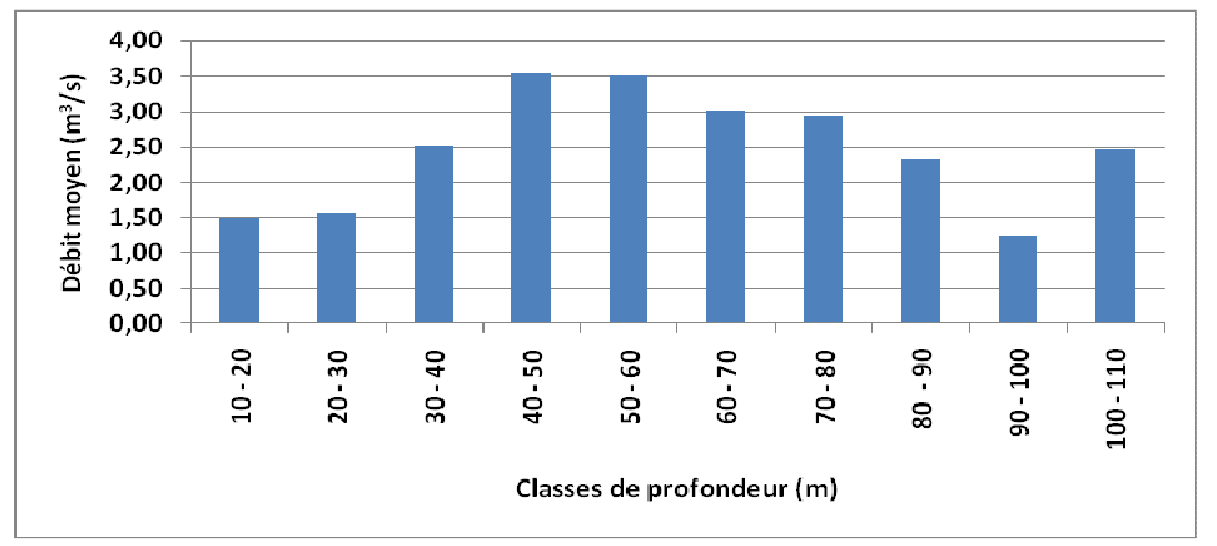

Figure 6 : Débit moyen en fonction des classes de profondeur. 


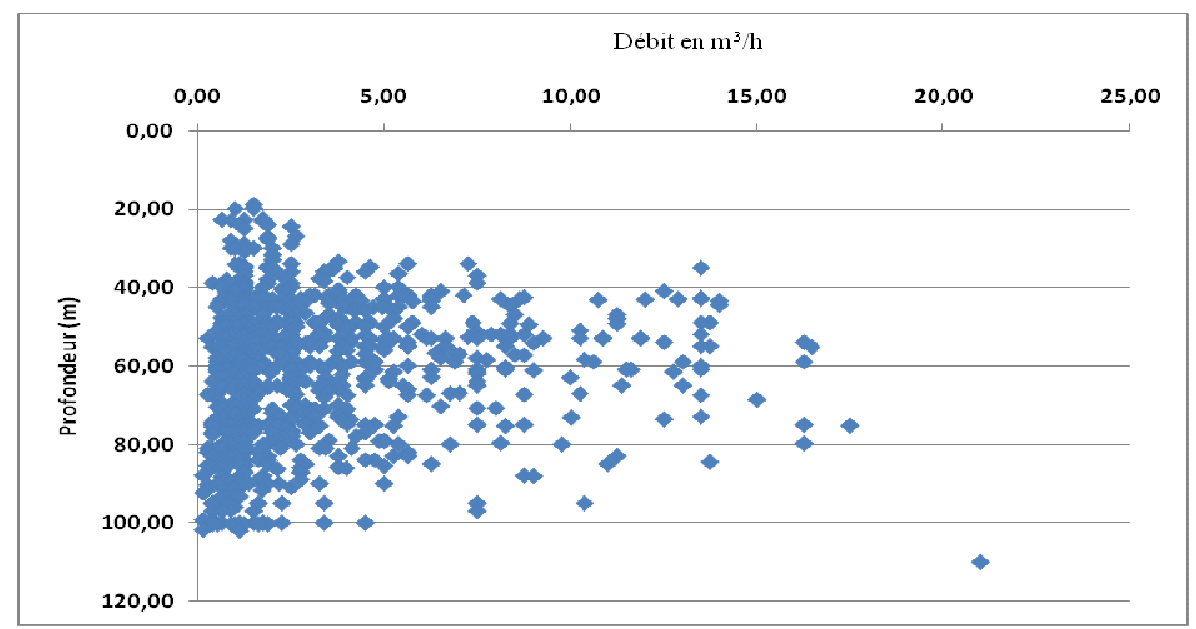

Figure 7 : Relation entre les profondeurs des forages et les débits.

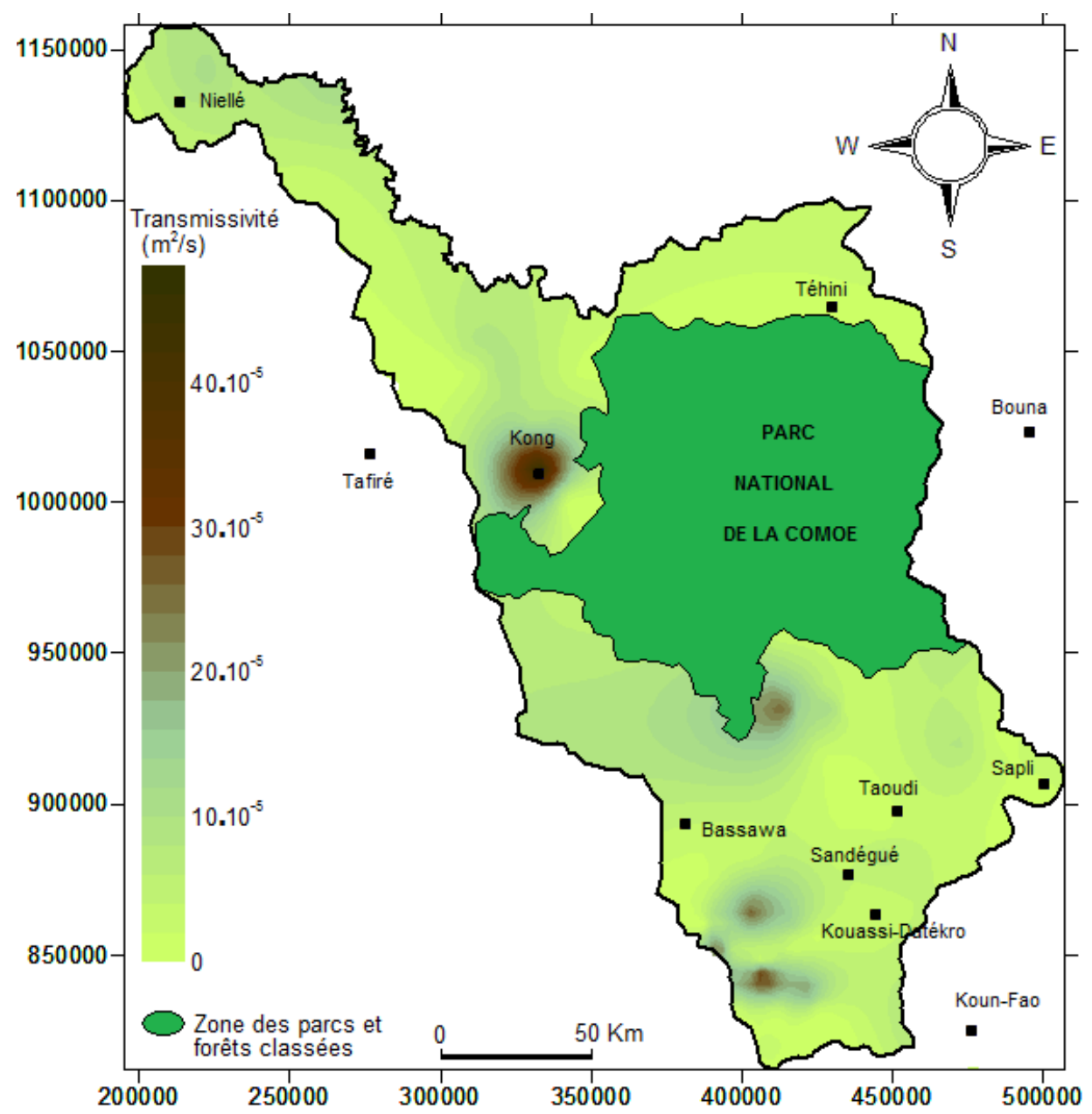

Figure 8: Carte de répartition des valeurs de transmissivité. 
Cela ne pourrait se passer autrement. En effet, la zone d'étude est située en climat tropical humide dans une zone de socle. Or ; sous un tel climat, le socle fissuré subit les effets de l'altération plus rapidement que sous un climat tempéré.

La grande taille des épaisseurs d'altération influence la profondeur des forages. En effet, $85,31 \%$ des forages ont une profondeur comprise entre $30 \mathrm{~m}$ et $90 \mathrm{~m}$. Pour ce qui concerne la productivité, les forages implantés sur les schistes donnent des débits plus importants (donc sont plus productifs) que ceux implantés sur les granitoïdes.

La zone fissurée qui est comprise entre la première et la dernière venue d'eau peut être utilisée pour caractériser la productivité des ouvrages, en plus du débit optimum (, Kouadio et al., 2008). Dans la zone de la haute Comoé, plus de $88 \%$ des arrivées d'eau sont comprises entre $20 \mathrm{~m}$ et $90 \mathrm{~m}$ et plus de $50 \%$ entre $30 \mathrm{~m}$ et $50 \mathrm{~m}$. Il ressort de l'analyse de la productivité des forages en fonction de la profondeur que les meilleurs débits pour l'hydraulique villageoise et l'hydraulique villageoise améliorée sont obtenus entre $40 \mathrm{~m}$ et $60 \mathrm{~m}$ de profondeur. Koita (2010) a mis en évidence sur le granite dans la région de Dimbokro-Bongouanou que la profondeur de $40 \mathrm{~m}$ est à la fois celle qui concentre les maxima de fréquence, de densité et de débit des arrivées d'eau.

Par ailleurs, plusieurs travaux réalisés sur le socle en Côte d'Ivoire ont mis en évidence que les meilleurs débits sont obtenus avec des forages ayant entre $30 \mathrm{~m}$ et $70 \mathrm{~m}$ de profondeur (Kouadio et al., 2008; Koita, 2010 ; Lasm et al., 2012). Néanmoins, des débits importants ont été obtenus à des profondeurs supérieures à $80 \mathrm{~m}$, même si le nombre de forages est faible. Même si au niveau de notre zone d'étude, le débit le plus important a été obtenu à une profondeur de $100 \mathrm{~m}$, il n'est pas très utile de réaliser des forages assez profonds pour l'hydraulique villageoise.

\section{Conclusion}

Cette étude a mis en relief la forte profondeur des forages dans le nord du bassin versant de la Comoé. De plus, les épaisseurs d'altération y sont importantes. Les forages implantés sur les schistes donnent des débits plus importants (donc sont plus productifs) que ceux implantés sur les granitoïdes. Les meilleurs débits pour l'hydraulique villageoise et l'hydraulique villageoise améliorée sont obtenus entre $40 \mathrm{~m}$ et $60 \mathrm{~m}$ de profondeur. Les valeurs de transmissivité calculées à partir des données d'essai de pompage varient de $5,59.10^{-6} \mathrm{~m}^{2} / \mathrm{s}$ à $2,01.10^{-4} \mathrm{~m}^{2} / \mathrm{s}$. Les résultats de cette étude sont intéressants pour minimiser les échecs pendant les campagnes d'implantation des forages.

\section{REFERENCES}

Adiaffi B. 2008. Apport de la géochimie isotopique, de l'hydrochimie et de la télédétection à la connaissance des aquifères de la zone de contact "soclebassin sédimentaire" du sud-est de la Côte d'Ivoire. Thèse de doctorat en Sciences de la Terre, Université Paris Sud, p. 231.

Anonyme. 1998. Schéma directeur pour le développement de la région du Zanzan. Haut-Commissariat au Développement des Régions de la Savane du Centre et du Nord, p. 97.

Biémi J, Jourda JP, Deslandes S, Gwyn H. 1997. Positionnement, productivité et gestion des forages en milieu fissuré de Côte d'Ivoire par télédétection et système d'information géographique. Atelier de la FAO sur Télédétection et ressources en eau, Montpellier. Séries Water report 16. (www.fao.org/docrep/w7320b18.htm).

JICA. 2001. Plan directeur de gestion intégrée des ressources en eau en République de Côte d'Ivoire, rapport principal. JICA, p. 446.

Goné DL. 2001. Contribution de l'étude des paramètres physico-chimiques des eaux souterraines à la compréhension du fonctionnement des systèmes 
hydrauliques en milieu fissuré de la région semi-montagneuse de Man (Ouest de la Côte d'Ivoire), Thèse de doctorat $3^{\text {ème }}$ cycle, Université Abobo-Adjamé (Côte d'Ivoire), 179p.

Koita M. 2010. Caractérisation et modélisation du fonctionnement hydrodynamique d'un aquifère fracturé en zone de socle. Région de DimbokroBongouanou (Centre Est de la Côte d'Ivoire). Thèse de doctorat, Université de Montpelier II, P. 235.

Kouadio KE, Savane I, Lasm T, Biemi J. 2008. Hydrogeology prospecting in crystalline and metamorphic area by spatial analysis of productivity potential. European Journal of Scientific Research, 22(3): 373-390.

Kouamé KJ. 2007. Contribution à la Gestion Intégrée des Ressources en Eaux (GIRE) du District d'Abidjan (Sud de la Côte d'Ivoire) : Outils d'aide à la décision pour la prévention et la protection des eaux souterraines contre la pollution. Thèse de doctorat, Université de Cocody, p. 252.

Kouamé F. 1999. Hydrogéologie des régions de montagne : apports des données de télédétection et des méthodes statistique et fractale a l'élaboration d'un système d'information hydrogéologique à référence spatiale des aquifères discontinus du secteur Man-Danané (Ouest de la Côte d'Ivoire). Thèse de doctorat $3^{\text {ème }}$ cycle, Université de Cocody, p. 235.

Lasm T, Fossou R, N'Guessan M, Onetie OZ, Baka D, Youan TM, Oga MS, Soro N. 2012. Contribution hydrogéologique à la connaissance des aquifères discontinus du département de ferké (nord de la Côte d'Ivoire) pour une meilleure alimentation en eau potable. Rev. Ivoir. Sci. Technol., 19: $114-135$.

Lasm T. 2000. Hydrogéologie des réservoirs fracturés de socle : analyses statistique et géostatistique de la fracturation et des propriétés hydrauliques. Application à la région des montagnes de Côte d'Ivoire (Domaine archéen). Thèse de doctorat Université de Poitiers, France, p. 273.

Lasm T. Kouame F. Soro N. Jourda JPR, Biémi J. 2004. Analyse géostatistique de la fracturation extraite de l'imagerie spatiale aéroportée et satellitaire. Application a la région de Man-Danané (Ouest de la Côte d'Ivoire). Rev. Ivoir. Sci. Technol., 05 : 135 - 154.

Savané I. Coulibaly K, Gion P. 2001. Variabilité climatique et ressources en eaux souterraines dans la région sémimontagneuse de Man. Sécheresse, 12(4): 231-237.

Touré S. 2005. Pétrologie et géochronologie du massif de granitoïde de Bondoukou (Nord-Est de la Côte d'Ivoire) : Evolution magmatique et contexte géodynamique au Protérozoïque inférieur (Paléoprotérozoïque); relations avec les formations volcaniques et volcanodétritiques du Zanzan; implications paléogéographiques. Thèse de doctorat d'Etat ès Sciences de la Terre, Université d'Abobo-Adjamé, p. 405. 